\title{
ARTICLE
}

Cite this: DOI: $10.1039 /$ xoxxooooox

Received ooth January 2017,

Accepted ooth January 2017

DOI: 10.1039/xoxxooooox

www.rsc.org/

\section{Structural disorder, filament growth and self- poisoning in short rods confined onto a flat wall}

\author{
Yongxiang Gao ${ }^{* a b}$, Violet Farkas $^{b}$, Roel. P. A. Dullens ${ }^{b}$ and Dirk G. A. L. Aarts $^{b}$
}

Confocal microscopy was used to directly observe the structural coarsening of the first layer of short colloidal rods sedimented onto a flat wall. Based on an image analysis algorithm we devised, quantitative information on the location, orientation and length of each particle can be extracted with high precision. At high density the system undergoes structural arrest, and becomes trapped in a disordered state of randomly arranged filaments that are composed of side-by-side aligned rods. The frustration of structural order is signalled by a new peak that emerges in the radial distribution function. Configuration analysis shows that the peak is primarily due to pairs of particles that are arranged in a " $T$ " shape, a configuration that is compatible with neither crystallization nor filament growth. Our results point to a selfpoisoning mechanism for the frustration of structural order, and highlight the importance of particle shape in controlling colloidal assembly thus materials properties.

\section{Introduction}

Dense assemblies of nonspherical hard particles are of considerable interests for both fundamental ${ }^{1}$ and applied ${ }^{2}$ research. Nonspherical particles demonstrate rich physics as well as packing configurations through entropic competition between the orientational and the translational degrees of freedom. For example, particles with low-degree of anisotropy show higher efficiency in randomly filling space than spheres ${ }^{3,4}$, and form glasses with stronger mechanical properties ${ }^{5}$; spherocylinders display various liquid crystalline phases ${ }^{6-8}$, depending on the aspect ratio; polyhedral have emerged as new building blocks for designing materials with controlled physical properties ${ }^{9}$.

Nonspherical particles confined to 2D have attracted an even larger body of research due to their topological simplification. Computer simulations have uncovered phase diagrams ${ }^{10,11}$, packing behaviours of ellipses ${ }^{12}$, and predicted the emergence of liquid crystalline phases in $2 \mathrm{D}$ spherocylinders ${ }^{13}$ and a tetratic phase in rectangles ${ }^{14}$. Experimentally, confining colloids onto a flat wall provides a natural and simple way to prepare $2 \mathrm{D}$ systems. It has been shown that kite-like platelets can significantly frustrate crystallization ${ }^{15}$ due to their shape; colloidal squares have revealed crystal structures with new symmetries $^{16}$. Yet, our understanding on the impact of shape on the self-assembled structures in 2D is still underdeveloped.

Here we investigate the structural behaviours of short rods confined onto a flat wall by gravity. Note that the particles have additional freedom of changing their orientation from vertical to horizontal. Combining confocal microscopy with image analysis, we follow the structural evolution at the single particle level as the system gradually increases its packing fraction during sedimentation. We aim to understand the interplay between the particle shape and ordering process, relevant for assembly in colloidal systems in general.
We will start by describing the details on the materials and methods in Section 2, including particle synthesis (2.1), experimental setup (2.2), and image analysis (2.3). We then present the results and discussion in Section 3. In the end, we summarize our conclusions in Section 4.

\section{Materials and methods}

\subsection{Particle synthesis}

Seed rods are first synthesized via a one-pot synthesis technique introduced by Kuijk et $a l^{17}$. Specifically, $30 \mathrm{~g}$ of Polyvinylpyrolidone (PVP, $\mathrm{M}_{\mathrm{w}}=40,000 \mathrm{~g} / \mathrm{mol}$; Sigma Aldrich) was

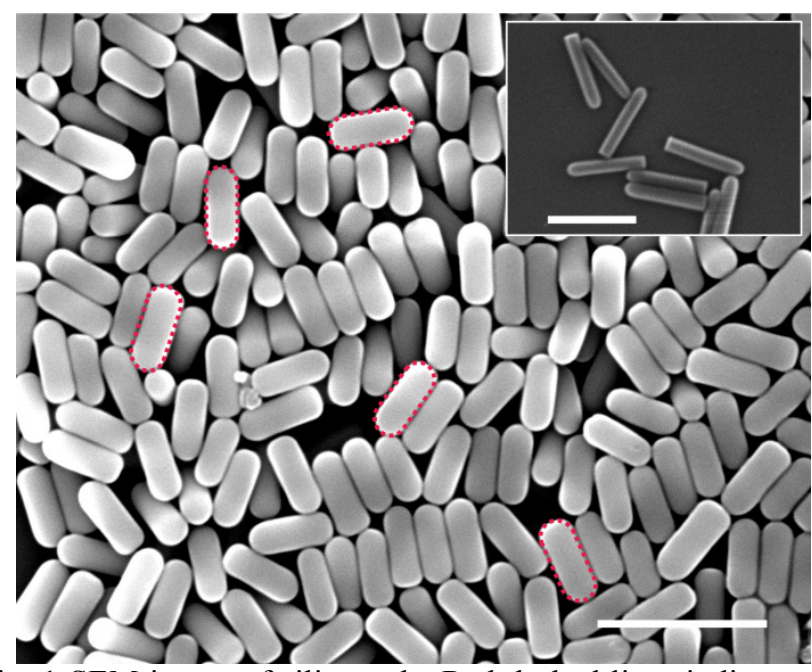

Fig. 1 SEM image of silica rods. Red dashed lines indicate the shape of spherocylinders. Initial seed rods are shown in the inset. Scalebars are $5 \mu \mathrm{m}$ (main figure) and $2 \mu \mathrm{m}$ (inset), respectively. 
dissolved in $300 \mathrm{ml}$ of 1-pentanol (Sigma Aldrich) by sonication (VWR ultrasonic cleaner) in a $500 \mathrm{ml}$ round-bottomed flask. Then, $8.1 \mathrm{ml}$ of deionized (DI) water (Millipore, $18.2 \mathrm{~m} \Omega$ ) and $1.8 \mathrm{ml}$ of $0.18 \mathrm{M}$ sodium citrate (Fisher Scientific) solution were added. The resulting mixture was shaken to form a water-in-pentanol emulsion. Then, $27 \mathrm{ml}$ of ethanol (Sigma Aldrich) and $6.075 \mathrm{ml}$ of ammonia solution ( $25 \mathrm{w} \%$; Merck Chemicals) were added, followed by shaking the solution to promote mixing. In the end, $1.8 \mathrm{ml}$ of tetraethyl orthosilicate (TEOS, 98\%, Acros Organics) was added to start the hydrolysis and condensation reactions for directional growth of silica. The mixture was left undisturbed overnight to react.

After the reaction was finished, the solution was centrifuged at 3500 RPM (Eppendorf centrifuge 5810) for 70 minutes and the supernatant was disregarded. To remove residue chemicals, the sediment was repeatedly washed in ethanol and water, following a pattern of ethanol (2 times)-water (3 times)-ethanol (2 times). The initial silica seeds are of bullet shape, and are approximately $2 \mu \mathrm{m}$ long and 300-400 nm wide (Fig. 1 inset), checked by a scanning electron microscope (SEM; Joel JSM-6010LV). Additional silica shells were further grown onto the particles to increase their size. Specifically, seed particles were dispersed in $100 \mathrm{ml}$ of ethanol in a $500 \mathrm{ml}$ flask. Under magnetic stirring, $10.8 \mathrm{ml}$ of ammonia solution, $9 \mathrm{ml}$ of DI water, and $0.6 \mathrm{ml}$ of TEOS were added. The reaction was left to proceed overnight (for a minimum of 6 hours). The resulted particles were washed in the same manner as described above. This whole procedure was repeated a further three times, and each time the rods were examined by SEM to check for size and aggregation. Their final length (L) and diameter (D) measured from $\sim 100$ particles are $2.68 \pm 0.16 \mu \mathrm{m}$ and $0.99 \pm 0.05 \mu \mathrm{m}$, respectively. The shape of the particles can be well approximated as spherocylinders, see Fig.1. Lastly, the particles were dried and kept in powder format for later use.

\subsection{Experimental setup}

A stock solution of the particles was prepared at a volume fraction of $\phi \sim 0.001$ by dispersing a weighted amount of dried silica rods in dimethylformamide (DMF, Acros Organics). DMF has viscosity of $0.92 \mathrm{mPa} \cdot \mathrm{s}$ at $20{ }^{\circ} \mathrm{C}$ and a refractive index (1.43) close to that of silica (1.45). The Brownian time of the particle is estimated to be $\sim 5$ seconds. A small amount of dye (Rhodamine B Isothiocyanate; Sigma Aldrich) was added to the solution to facilitate fluorescence confocal imaging. Silica rods have a higher density $(1.9 \mathrm{~g} / \mathrm{ml})$ than $\operatorname{DMF}(0.944 \mathrm{~g} / \mathrm{ml})$, and the gravitational length of the particles is

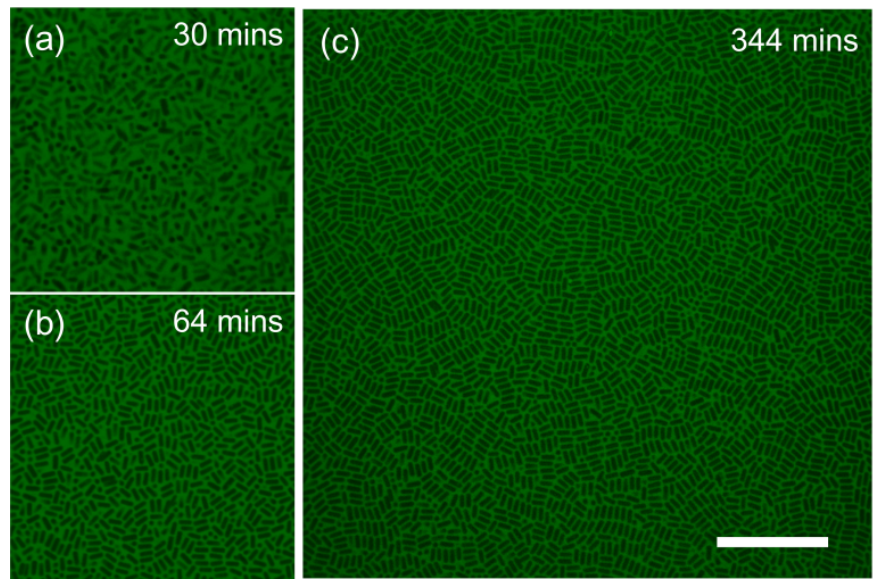

Fig. 2 Confocal images of the first layer of the sample. Representative images are shown for (a) $t=30$, (b) 64 and (c) 344 minutes after the sample was prepared and placed on the microscope stage. Scalebar represents $20 \mu \mathrm{m}$.

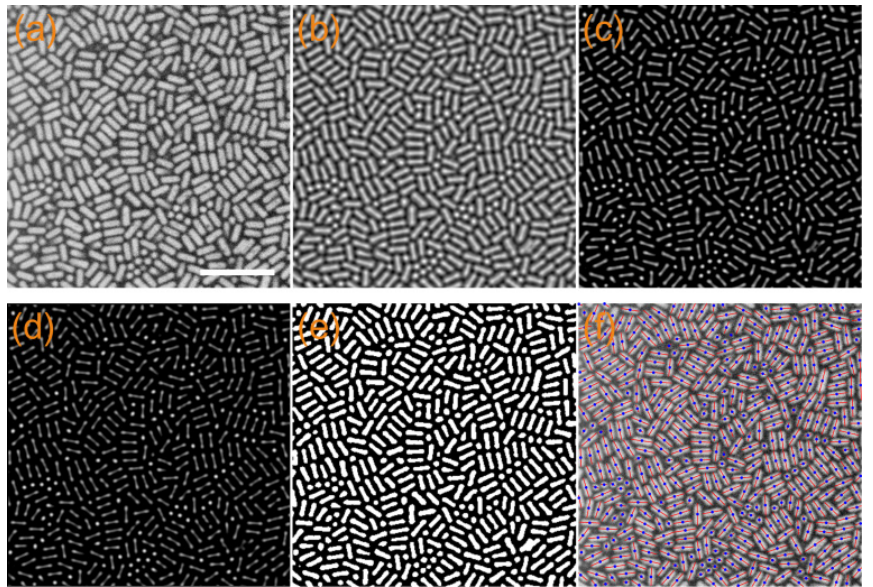

Fig. 3 Steps for image analysis. (a) Inverted raw image; (b) Optimized correlation map that best resembles the raw image; (c) Cleaned correlation map by removing pixels with correlation coefficient lower than a $\mathrm{c}_{\text {cut }}$ of 0.1 ; (d) The resulted image after applying a bandpass filter for further noise and background removal ${ }^{18}$. (e) Binarized image of (d); (f) Overlaying results onto the inverted raw image, with the centroids marked by blue dots, and projection length and particle orientation represented by red lines.

estimated to be $0.24 \mu \mathrm{m}$ and the gravitational Peclet number is estimated to be $\sim 2$. $60 \mu \mathrm{l}$ of the stock solution was loaded to a homebuilt cylindrical sample chamber of $1 \mathrm{~cm}$ tall and $0.6 \mathrm{~cm}$ wide (inner diameter), glued to a piece of coverslip. The top of the sample chamber is sealed with taflon tape.

Particles sediment slowly to the coverslip under gravity. The area fraction of the first layer on the coverslip increases with time, and the system gradually approaches an arrested state. Eventually, there is a second diffusive and not-well-defined layer on top of the first layer. To directly visualize the structure evolution of the system, we use a confocal laser scanner (Thorlabs) mounted on an inverted light microscope (Olympus IX73) equipped with a $60 \mathrm{X}$ oil-immersion objective lens (PLAPON; NA=1.42). The excitation wave length was set at $532 \mathrm{~nm}$. The microscope detects the excited dye in the background solvent, while the colloids remain dark, see Fig. 2. Typically, images of $1024 \times 1024$ pixels $^{2}$, corresponding to an imaging area of $102.4 \times 102.4 \mu^{2}$, were taken at 30 -second intervals.

\subsection{Image analysis}

To extract quantitative information on the structure of the system at the single-particle level, we devise an image analysis algorithm to identify the centroid, orientation and length of particles. The algorithm includes the following steps: (1) Invert the original image so that particles appear bright on a dark background (Fig. 3a); (2) Identify a Gaussian-like template texture that best resemble features in the image and compute the correlation coefficient map ${ }^{19}$, whose value essentially represents the probability of finding a point-like feature (Fig. 3b); (3) Remove pixels from the correlation map whose value is below a threshold value $c_{\text {cut }}$ (Fig. 3c); (4) Running a bandpass filter ${ }^{18}$ to further remove noise and enhance contrast (Fig. 3d); (5) Binary the image by using an intensity threshold $\mathrm{I}_{\text {cut }}$ (Fig. 3e); (6) Using the built-in function "regionprops" of MatLab to compute the centroid, length, and orientation of each rod; the results are overlaid on top of the inverted image for checking the quality of the image analysis algorithm (Fig. 3f). We estimate the spatial resolution to be $\sim 1 / 4$ of a pixel in each dimension and $\sim 0.03 \mathrm{rad}$ in the orientation. 


\section{Results and discussion}
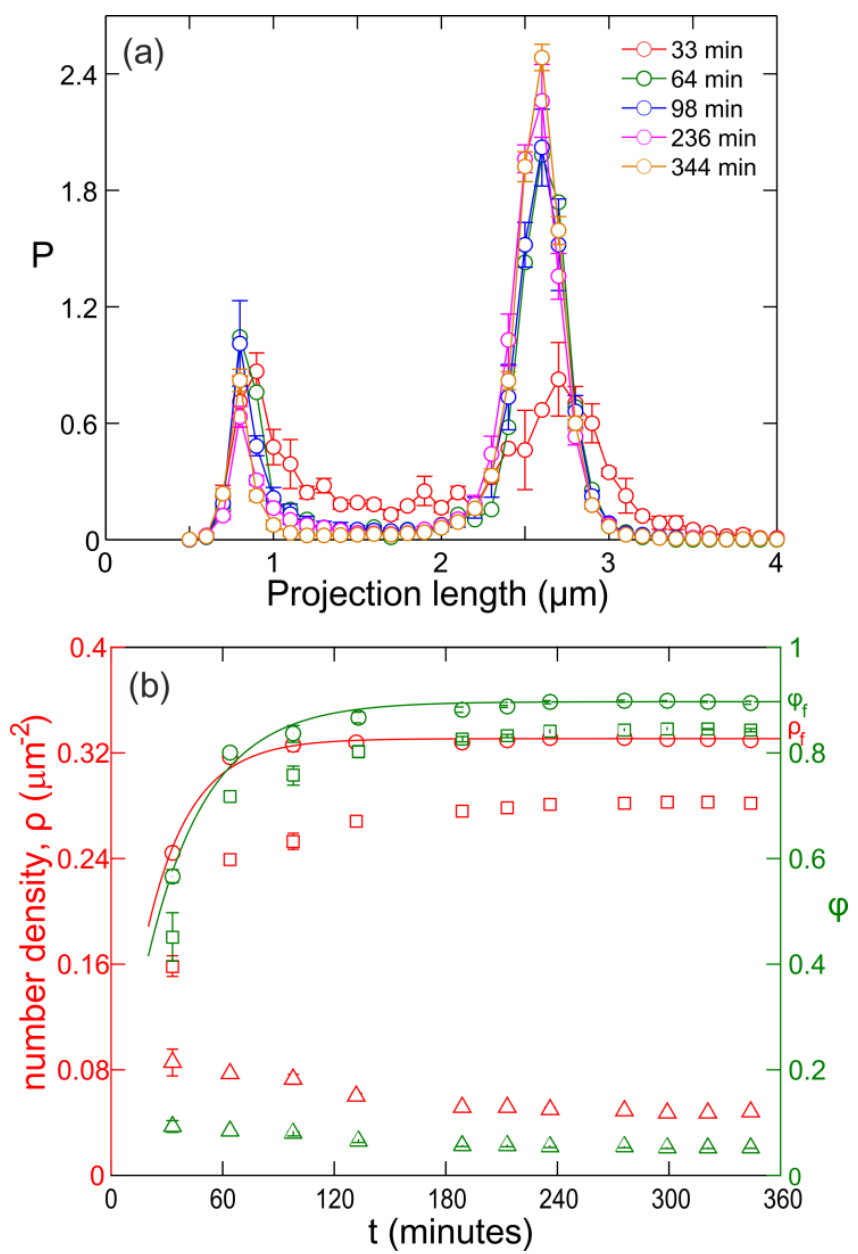

Fig. 4 Densification of experimental system with time. (a) Probability distribution of the projection lengths of rods at various times. (b) The number density and the area fraction as a function of time for all the rods (circles), horizontal rods (squares) and vertical rods (triangles). The solid lines are fits to the experimental data with $\rho=0.331 \times[1-\exp (-\mathrm{t} / 23.8)]$ (red line) and $\varphi=0.897 \times[1-\exp (-\mathrm{t} / 32.0)]$ (green line), respectively.

In Fig. 4a, we present the probability distributions of the projection lengths of all the particles at various times, where $t=0$ represents when the sample is placed on the microscope stage. We found that the distributions are bimodal, peaked at $\mathrm{L}_{\mathrm{p}} \sim 0.8 \mu \mathrm{m}$ and $\mathrm{L}_{\mathrm{p}} \sim 2.6 \mu \mathrm{m}$, respectively. These two lengths are close to the diameter and length of the rods measured by SEM, and we attribute the difference to the inaccuracy of measuring particle size with optical microscopy and image analysis. This implies that the particles prefer either standing up or lying flat on the surface. This is also clear when we examine confocal images shown in Fig. 2. Note that the measured length is merely used to separate the two populations and its inaccuracy has negligible influence on the result.

We separate the two populations by setting a threshold length of $1.5 \mu \mathrm{m}$, considering a particle to be a disk $\left(\mathrm{L}_{\mathrm{p}}=\mathrm{D}\right)$ if $\mathrm{L}_{\mathrm{p}} \leq 1.5$ $\mu \mathrm{m}$ and a rod with the full length $\left(\mathrm{L}_{\mathrm{p}}=\mathrm{L}\right)$ if $\mathrm{L}_{\mathrm{p}}>1.5 \mu \mathrm{m}$. Over time, the percentage of horizontal rods (lying flat) increases and that of vertical rods (standing up) decreases, see Fig. 4a. We further examine the number density $(\rho)$ for all the particles and the two populations separately in Fig. $4 b$. We find that the
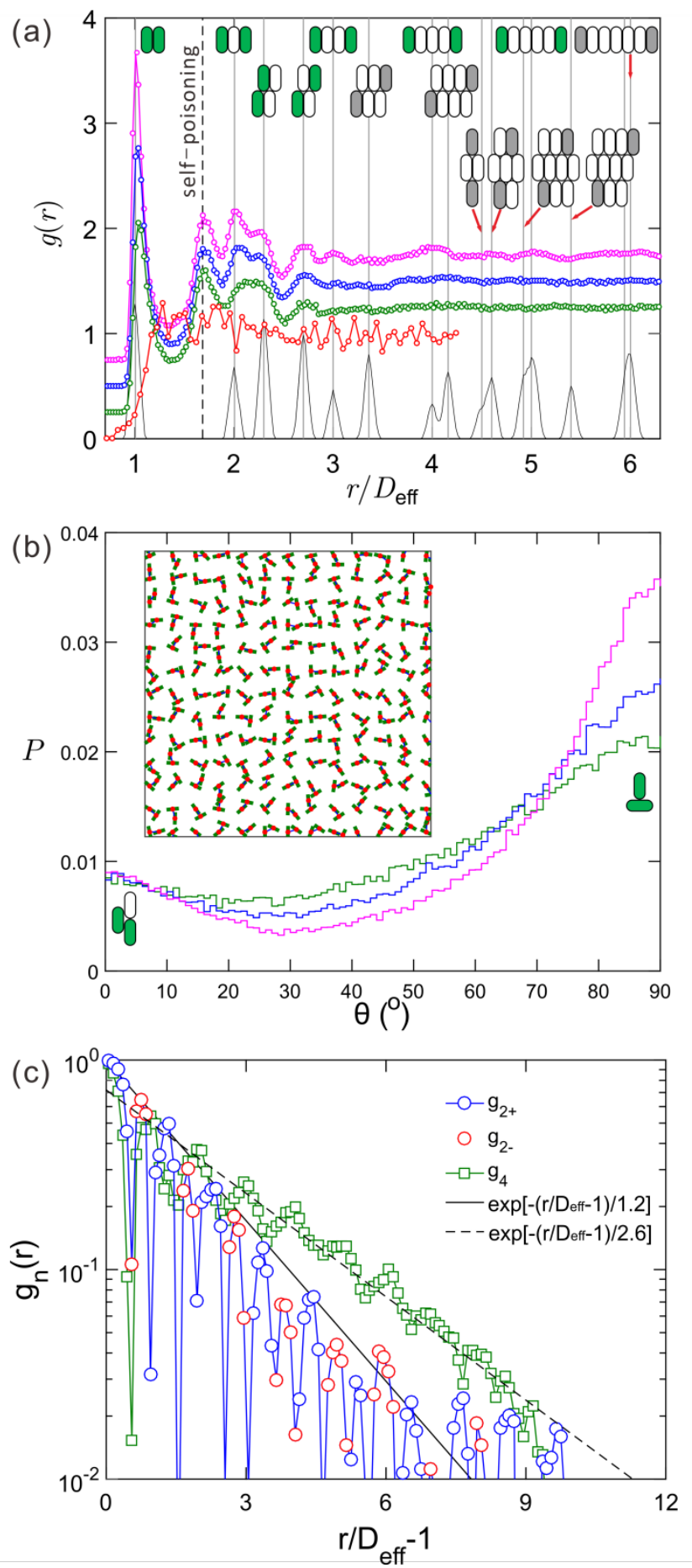

Fig. 5 Structural frustration by self-poisoning. (a) RDFs at $\varphi=0.567$ (red), 0.836 (green), 0.867 (blue), and 0.897 (purple). The locations of expected peaks in a perfect crystal (gray solid lines) and RDF of a perfect crystal with $10 \%$ added noise (black solid line) are also shown for comparison. Inset: configurations of particle pairs shown up (green) or being absent (gray) in the experiments. The location of the "self-poisoning" peak is also shown (black dashed line). (b) Histograms of angles formed by particle pairs in the self-poisoning peak at $\varphi=0.836$ (green), 0.867 (blue), and 0.897 (purple). Inset: a subset of randomly chosen pair configurations at $\varphi=0.897$. (c) Orientational correlation functions of $g_{2}$ and $g_{4}$ together with the fits. At certain separations, $g_{2}$ shows negative values or anti-correlation, which is inverted for display (red symbols). 
number density of vertical rods decreases with time despite the increase in number density for all the particles. This indicates that the increase in the population of horizontal particles has two contributions: one comes from the increasing number of particles reaching to the first layer; the other comes from the decrease in the vertical population. The total number density reaches to its final value $\left(\rho_{\mathrm{f}}\right)$ at $\sim 130$ minutes, which is $\sim 100$ minutes earlier than the time at which the number densities of standing rods or flat rods reach a plateau. The population of vertical rods decreases with time due to the gravitational potential; yet at long times this no longer happens as it becomes increasingly difficult to make more room for a horizontal rod, i.e. the existence of a small fraction of standing rods may be ascribed to their higher efficiency for void filling ${ }^{20,21}$.

Our silica rods carry negative surface charges. We therefore map the particles' dimensions to effective hard rods by matching the peak positions in the radial distribution function that correspond to the side-by-side separation and the shortest separation of rods between two different layers at the final density (see Fig. S1 and the Supplementary information). From this mapping, we obtain the effective diameter $\left(\mathrm{D}_{\text {eff }}\right)$ and length $\left(\mathrm{L}_{\text {eff }}\right)$ of the particles to be 1.18 $\mu \mathrm{m}$ and $2.80 \mu \mathrm{m}$, respectively. Based on $\mathrm{D}_{\text {eff }}$ and $\mathrm{L}_{\text {eff, }}$, we can more accurately calculate the packing fraction $(\varphi)$ of the system,

$$
\varphi=\rho_{\mathrm{L}}\left(\frac{\pi \mathrm{D}_{\mathrm{eff}}^{2}}{4}+(\mathrm{L}-2 \mathrm{D}) \mathrm{D}_{\mathrm{eff}}\right)+\rho_{\mathrm{S}}\left(\frac{\pi \mathrm{D}_{\mathrm{eff}}^{2}}{4}\right) .
$$

Here, $\rho_{\mathrm{L}}$ and $\rho_{\mathrm{S}}$ are the number density of the horizontal rods and vertical rods, respectively. The area fractions as a function of time for all the particles and the two subpopulations are plotted in Fig. 4b. The total area fraction reaches to its final value $\left(\varphi_{\mathrm{f}}\right)$ of 0.897 at $\mathrm{t} \sim 270$ minutes, and no further increase is observed for over 1 hour. Note that $\varphi_{\mathrm{f}}$ is well beyond the freezing and the melting points of hard spherycylinders ${ }^{13}$ with an aspect ratio that corresponds to the effective dimensions of our particles, which are $\sim 0.797$ and $\sim 0.836$, respectively. In the end, the area fraction taken by the vertical rods is small, $\sim 5 \%$.

To characterize the structure of the system, we compute the radial distribution function (RDF), see Fig. 5a. At low density $(\varphi \leq 0.567)$, there is hardly any structural correlation observed. With increasing area fraction, the correlation becomes both stronger and extends over longer distances. We find that the system prefers to form small compact structures at first. This is shown by the RDF at $\varphi=0.836$, where the system has developed peaks corresponding to particles belonging to two different layers but not a peak at $r / D_{\text {eff }}=3$, which corresponds to four particles in the same layer. Interestingly, we observe that with a further increase in the area fraction, more peaks have been developed for particles belonging to the same layer, but no further peaks developed for particles belonging to two or more layers. In other words, the system favors the growth of filament-like structures of one layer thickness over multiple layers. Similar behaviour has been observed in computer simulations of short rods in $3 \mathrm{D}^{22}$. In addition, we discover a new peak at $r / D_{\text {eff }}$ of 1.68 , which is absent in a perfect crystal. We examine the configurations of particle pairs corresponding to the new peak, with a randomly selected subset shown in Fig. $5 \mathrm{~b}$ (inset). We find that most of the particle pairs are arrange in a ' $\mathrm{T}$ ' shape. This is also evident in the angular probability distribution, which shows a strong peak at $90^{\circ}$ (Fig. 5b), suggesting a local tetratic order. We have further analyzed the orientational correlation functions ${ }^{13} \mathrm{~g}_{2}(\mathrm{r})=\langle\cos [2(\theta(0)-\theta(\mathrm{r}))]\rangle$ and $\mathrm{g}_{4}(\mathrm{r})=\langle\cos [4(\theta(0)-\theta(\mathrm{r}))]\rangle$, shown in Fig. 5c. Both functions decay exponentially with separation. We observe no long range tetratic order, indicating that no tetratic phase is formed. Our result may help to resolve contradictive observations that have been made in computer simulations ${ }^{13,23}$. The ' $\mathrm{T}$ ' structure is clearly incompatible with crystallization, and is a 2D analogue of the self-poisoning effect observed in simulated 3D short rods ${ }^{22}$. We therefore label the new peak in the RDF as a selfpoisoning peak.

Since the system does not favour growing into multiple layers, we then perform 1-d filament analysis. If the separation (r) and angle $(\theta)$ between a pair of particles satisfy the following two criteria, $\mathrm{r} \leq 1.7 \mu \mathrm{m}$ and $\theta \leq 30^{\circ}$, they are considered to belong to the same filament. In Fig. 6 a, we show the result of filament decomposition for a configuration in the final state. Colors are coded such that no neighboring filaments have the same color. For completeness, cluster decomposition is also done for standing rods independently based on a single separation criterion, $\mathrm{r} \leq 1.62 \mu \mathrm{m}$, where $1.62 \mu \mathrm{m}$ corresponds to the location of the first minimum in the RDF.

The probability distribution of a filament to have a length of $N$ particles, $P(N)$, follows an exponential distribution, as shown in Fig. 6b. The mean filament length, $<N_{\mathrm{c}}>=\sum N^{2} P(\mathrm{~N}) / \sum N \mathrm{P}(N)$, first grows with time (or area fraction) and then becomes

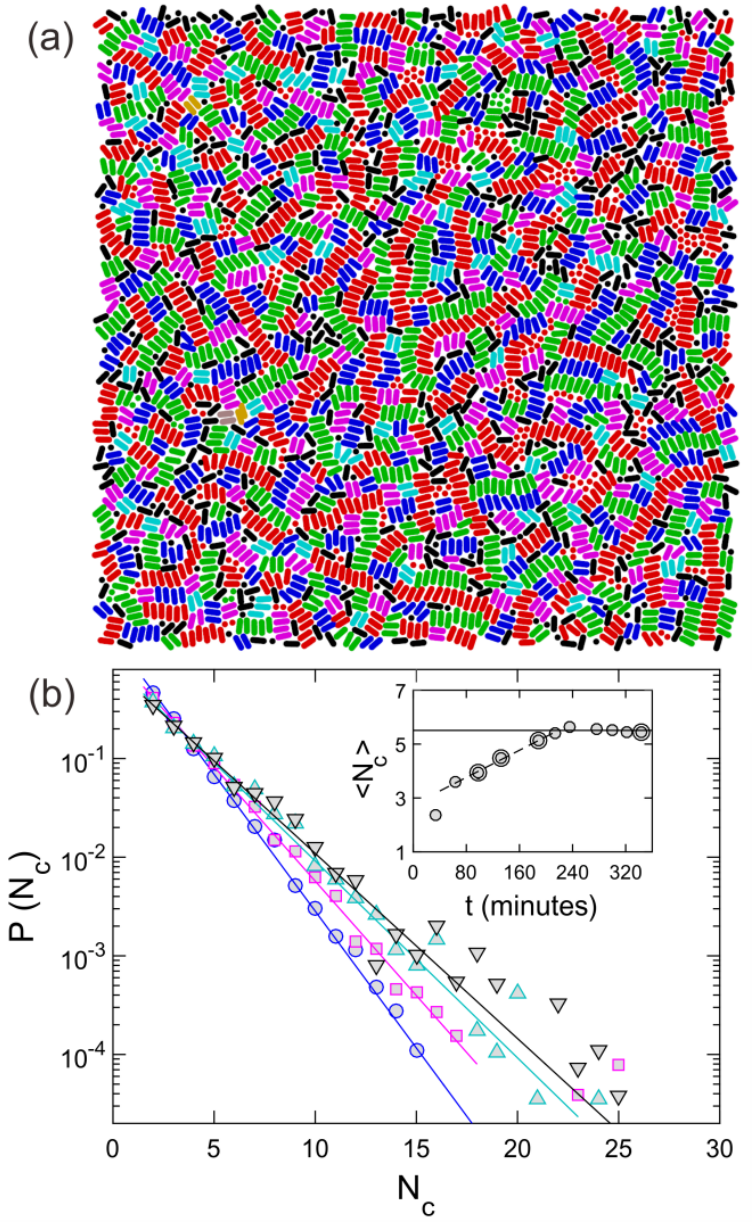

Fig. 6 Filament decomposition and growth. (a) Filament decomposition for a configuration at $\varphi=0.897$. Colors are assigned to filaments to distinguish neighboring ones. Singlet particles are shown as black. (b) Filament length distributions at $\varphi=0.836$ (circles), 0.867 (squares), 0.882 (upright triangles), 0.897 (inverted triangles). Inset: the dependence of average filament size with time, with the four experimental points shown in the main panel highlighted by larger circles. 
arrested. The length of the filament continues to grow with area fraction, or if there is still room to add in more particles or allow particles to change configuration from vertical to horizontal. When the system reaches its final packing fraction, no further structural coarsening is observed. Eventually, the system is kinetically trapped in a highly disordered state of randomly placed filaments of particles. Note that dense packing of identical hard disks tends to form a hexagonally packed crystal due to the lack of structural frustration ${ }^{24}$. As such, our results highlight the importance of shape in self-frustrating crystallization. Interestingly, the disordered dense arrested structure has an area fraction (0.897) much higher than the random close packing of polydispersed disks (0.839), and close to the maximum density of random close packing achievable with ellipses $(0.895)^{12}$. This again illustrates the impact of shape anisotropy for improving the density of random close packing $^{3,4}$.

\section{Conclusions}

In summary, we have performed experimental study of monodispersed colloidal short spherocylinders confined onto a flat wall by gravity. A new image analysis algorithm is devised to extract quantitative information for rodlike particles in high precision. We find that the shape anisotropy make the system self-trapped in a disordered state, which is composed of randomly placed $1-\mathrm{d}$ filaments. The failed crystal phase can be explained by the tendency of the system to form ' $T$ ' structures, which act as a poison for both crystallization and filament growth. We speculate that the self-poisoning effect will depend on the particles' aspect ratio: for rods with a much lower aspect ratio, the shape effect may not be strong enough to disturb the emergence of crystalline order, resulting in a plastic crystal due to the rotational freedom of particles; for rods with a much larger aspect ratio, the strong alignment effect from neighboring particles may lead to the formation of a nematic phase or other more ordered phases without self-poisoning at high density. Our results highlight the importance of shape in self-frustrating crystallization for materials assembly, which may stimulate more studies on exploiting shape for the design of superior glass formers ${ }^{15,25}$.

\section{Acknowledgements}

Y.G. acknowledges financial support from Marie Curie actions (FP7-PEOPLE-2012-IIF No. 327919) and Shenzhen University.

\section{Notes and references}

${ }^{a}$ Institute for Advanced Study, Shenzhen University, Nanshan District, Shenzhen, Guangzhou, China, 518060

${ }^{b}$ Department of Chemistry, Physical and Theoretical Chemistry Laboratory, University of Oxford, South Parks Road, Oxford, OX1 3QZ, UK

*Address correspondence to yongxiang.gao@szu.edu.cn.

$\dagger$ Electronic Supplementary Information (ESI) available: [Mapping experimental system to the hard rod based on radial distribution function]. See DOI: $10.1039 / \mathrm{b} 000000 \mathrm{x} /$

1 S. Torquato and F. H. Stillinger, Rev. Mod. Phys., 2010, 82, 2633-2672.

2 S. C. Glotzer and M. J. Solomon, Nat. Mater., 2007, 6, 557-562.

3 S. R. Williams and A. P. Philipse, Phys. Rev. E, 2003, 67, 051301.

4 A. Donev, I. Cisse, D. Sachs, E. Variano, F. H. Stillinger, R. Connelly, S.

Torquato and P. M. Chaikin, Science, 2004, 303, 990-993.
5 R. C. Kramb, R. Zhang, K. S. Schweizer and C. F. Zukoski, Phys. Rev. Lett., 2010, 105, 055702.

6 L. Onsager, Ann. N. Y. Acad. Sci., 1949, 51, 627-659.

7 P. Bolhuis and D. Frenkel, J. Chem. Phys., 1997, 106, 666-687.

8 A. Kuijk, D. V. Byelov, A. V. Petukhov, A. van Blaaderen and A. Imhof, Farad. Discuss., 2012, 159, 181

9 P. F. Damasceno, M. Engel and S. C. Glotzer, Science, 2012, 337, 453-457. 10 J. A. Cuesta and D. Frenkel, Phys. Rev. A, 1990, 42, 2126-2136.

11 W. S. Xu, Y. W. Li, Z. Y. Sun and L. J. An, J. Chem. Phys., 2013, 139, 024501 .

12 G. Delaney, D. Weaire, S. Hutzler and S. Murphy, Philos.Mag. Lett., 2005, 85, 89-96.

13 M. A. Bates and D. Frenkel, J. Chem. Phys., 2000, 112, 10034.

14 A. Donev, J. Burton, F. H. Stillinger and S. Torquato, Phys. Rev. B, 2006, 73,054109 .

15 K. Zhao and T. G. Mason, Proc. Natl. Acad. Sci. U.S.A., 2015, 112, 12063-12068.

16 K. Zhao, R. Bruinsma and T. G. Mason, Proc.Natl. Acad. Sci. U.S.A., 2011, 108, 2684-2687.

17 A. Kuijk, A. van Blaaderen and A. Imhof, J. Am. Chem. Soc., 2011, 133, 2346-2349.

18 J. C. Crocker and D. G. Grier, J. Colloid Interface Sci., 1996, 179, 298310.

19 Y. Gao and M. E. Helgeson, Opt. Express, 2014, 22, 10046.

20 M. Ramaioli, L. Pournin and T. M. Liebling, Phys.Rev. E, 2007, 76, 021304.

21 D. L. Blair, T. Neicu and A. Kudrolli, Phys. Rev. E, 2003, 67, 031303.

22 T. Schilling and D. Frenkel, Phys. Rev. Lett., 2004, 92, 085505.

23 Y.-S. Su and L. I, Phys. Rev. E, 2015, 92, 012319.

24 A. Donev, S. Torquato, F. H. Stillinger and R. Connelly, J. Appl. Phys., 2004, 95, 989-999.

25 Z. Zheng, F. Wang and Y. Han, Phys. Rev. Lett., 2011, 107, 065702. 